\title{
Correction to: Conceptualizing Multiplicities of Scientific Literacy from Five Theoretical Perspectives
}

\author{
Sophia Jeong, Gretchen King, David Pauli, Cary Sell, and David Steele
}

\section{Correction to:}

Chapter 1 in: T. W. Teo et al. (eds.), Science Education in the 21st Century, https://doi.org/10.1007/978-981-15-5155-0_1

In the original version of the book, in the following correction has been incorporated: The chapter author's "Sophia (Sun Kyung) Jeong" first and last name have been corrected. The correction chapter and book have been updated with the change. 